Article

\title{
Assessment of Lipophilicity Indices Derived from Retention Behavior of Antioxidant Compounds in RP-HPLC
}

\author{
Ioana Anamaria Sima ${ }^{1}$, Agata Kot-Wasik ${ }^{2}$, Andrzej Wasik ${ }^{2, *}$, Jacek Namieśnik ${ }^{2}$ \\ and Costel Sârbu ${ }^{1}$ \\ 1 Faculty of Chemistry and Chemical Engineering, Babeş-Bolyai University, Arany Janos Str., No. 11, \\ RO-400028 Cluj-Napoca, România; ioanatuhutiu@ymail.com (I.A.S.); csarbu@chem.ubbcluj.ro (C.S.) \\ 2 Faculty of Chemistry, Gdansk University of Technology, 80-233 Gdansk, Poland; \\ agawasik@pg.gda.pl (A.K.-W.); chemanal@pg.gda.pl (J.N.) \\ * Correspondence: wasia@pg.gda.pl; Fax: +4858-347-2694
}

Academic Editor: Isabel C. F. R. Ferreira

Received: 27 February 2017; Accepted: 27 March 2017; Published: 29 March 2017

\begin{abstract}
Reverse phase high pressure liquid chromatography was employed in order to evaluate the lipophilicity of antioxidant compounds from different classes, such as phenolic acids, flavanones, flavanols, flavones, anthocyanins, stilbenes, xantonoids, and proanthocyanidins. The retention time of each compound was measured using five different HPLC columns: RP18 (LiChroCART, Purosphere RP-18e), C8 (Zorbax, Eclipse XDBC8), C16-Amide (Discovery RP-Amide C16), CN100 (Saulentechnik, Lichrosphere), and pentafluorophenyl (Phenomenex, Kinetex PFP), and the mobile phase consisted of methanol and water ( $0.1 \%$ formic acid) in different proportions. The measurements were conducted at two different column temperatures, room temperature $\left(22^{\circ} \mathrm{C}\right)$ and, in order to mimic the environment from the human body, $37^{\circ} \mathrm{C}$. Furthermore, principal component analysis (PCA) was used to obtain new lipophilicity indices and holistic lipophilicity charts. Additionally, highly representative depictions of the chromatographic behavior of the investigated compounds and stationary phases at different temperatures were obtained using two new chemometric approaches, namely two-way joining cluster analysis and sum of ranking differences.
\end{abstract}

Keywords: antioxidants; lipophilicity; reversed phase high-pressure liquid chromatography (RP-HPLC); PCA; sum of ranking differences

\section{Introduction}

A powerful weapon in the fight against free radicals consists of the polyphenolic compounds which exert significant biological resistance against oxidants. Research is suggesting that both the classical antioxidant properties of the polyphenolic compounds (given by the hydrogen-donating capacity through their molecular structure) and their metal-chelating properties (effectively preventing transition metals from catalyzing oxidation reactions) may be important elements in the overall effectiveness of such compounds against free radical oxidations [1].

In order to benefit from the properties of the polyphenolic compounds, it would be of great interest to conduct quantitative structure-property relationship (QSPR) studies regarding their lipophilicity. This parameter represents the extent to which a substance prefers a hydrophilic or a lipophilic medium and the distribution between media with different polarities suggests the behavior of a compound in experimental, natural, or biologic environments. Thus, in the human body, a compound with a high lipophilicity index (hydrophobic) will be distributed mainly in lipid bilayers and those with a low lipophilicity index (hydrophilic) will be distributed mainly in blood and serum. Knowing that 
this property of a molecule is particularly useful when considering the administration of any kind of medication, because lipophilicity determines several parameters of drugs, such as the ability to reach its target, the affinity for the target, and how long it will remain active in the body.

The methods for determining lipophilicity were classified by Sangster [2] in two categories: direct methods, in which the compound is quantitatively determined in one or both phases, and indirect methods, which do not require a quantitative analysis. The most popular direct method is the "shakeflask" method, whereas the chromatographic techniques are recognized as indirect methods for the determination of lipophilicity.

Generally, the chromatographic methods are based on determining the retention parameters and the most used method is the reversed phase high-pressure liquid chromatography (RP-HPLC) [3,4]. The principles of determination were established by Snyder and Kirkland [5], and in HPLC the affinity of a solute for the stationary phase is characterized by the retention factor $(k)$, defined as $k=\left(t_{\mathrm{R}}-t_{0}\right) / t_{0}$, where $t_{\mathrm{R}}$ is the retention time and $t_{0}$ is the dead time.

It has been demonstrated experimentally that logk is linearly inter-correlated with the volume fraction of the organic co-solvent $(\phi, C)$, following the classic model of Soczewinski's and Snyder's equation [6]: $\log k=\log k_{w}-S \phi$, where $\log k_{w}$ is considered to be the chromatographic lipophilicity index and represents the retention factor for pure water as eluent, $S$ is widely associated with the solvent strength or with the sorbent specific surface area, and $\phi$ is the volume fraction of the organic modifier.

The direct measurement of $\log k_{w}$ is the often very difficult, if not impossible, due to the fact that it can lead to very long retention time and at the same time to excessive broadening of the peak. For this reason, measuring $\mathrm{k}$ with different ratios of water-organic solvent mixture as mobile phases is preferred, and the extrapolation of the correlation between logk vs. \% organic modifier indicates the value of $\log k$ when using only water as the mobile phase.

An alternative to the lipophilicity index is the so-called chromatographic hydrophobicity index or Valko's index $\phi_{0}[7,8]$, which represents the volume fraction of the organic solvent in the mobile phase for which the amount of solute in the mobile phase is equal to that in the stationary phase $(k=1$, $\log k=0$ ). This parameter is described using the equation: $\phi_{0}=\log k_{w} / S$. Furthermore, in the final years, new lipophilicity indices were obtained using principal component analysis (PCA). Thus, the score corresponding to the first principal component (PC1), obtained by applying PCA to the matrix formed by the retention parameters $(k$ and $\log k)$, has proven to give highly valuable information regarding the lipophilicity [3,4,9-12].

In addition, the continuous requirement of the pharmaceutical industry to have efficient methods to rapidly assess the lipophilicity of newly synthetized compounds, has made the use of computational approaches for the prediction of this parameter more popular. Their advantages arise out of the fact that they do not require any experimental work, which drastically reduces the costs. Thus, much computer software that calculates several lipophilicity descriptors estimated by different algorithms based on structural, topological, or property considerations has been developed [13]. Mannhold et al. [14] present, in their review, the state-of-the-art in the development of $\log \mathrm{P}$ prediction approaches and detailed description of the methodology background of the major categories: substructure-based methods (fragmental, atom-based) and property-based methods (empirical approaches, 3D structure based, topological descriptors) are also given.

Furthermore, in order to compare, classify, and determine the best experimental method or computational approach for the determination of lipophilicity, a novel method of assessment has recently been developed by K. Heberger, namely the sum of ranking differences (SRD) [15]. This methodology has been successfully applied for the comparison of calculated lipohilicity scales with indices derived from retention behavior on RP-HPLC and HPLC-hydrophilic interaction columns [16]; the comparison of lipophilicity measures obtained by typical RP-TLC experiments with in silico approaches and lipophilicity measures obtained by micellar chromatography and typical RP-TLC experiments combined with in silico approaches [17]; liphophilicity measures comparison with classical and novel chemometric methods [18]. 
In view of the above considerations, the aim of this study was to assess the lipophilicity indices derived from the retention behavior of antioxidant compounds, estimated on five different HPLC columns at two different temperatures, as well as to compare the experimental indices with those obtained through computational approaches.

\section{Materials and Methods}

$\mathrm{T}$ (7) caffeic acid, (8) vanillic acid, (11) chlorogenic acid, and (17) gentisic acid; flavonoids: flavanones: (3) naringenin, (4) naringin, (13) hesperetin, and (14) hesperidin, flavanols: (9) (+)-catechin, (10) (-)-epicatechin, and (15) (-)-epigallocatechin gallate, flavonols: (12) rutin, and flavones: (18) apigenin; anthocyanins: (16) pelargonidin; stilbenes: (19) pterostilbene and (21) resveratrol; xantonoids: (20) mangiferin; proanthocyanidins: (22) C1 type proanthocyanidin. For each compound, solutions in concentration of $1 \mathrm{mg} / \mathrm{mL}$ in methanol were prepared using HPLC grade standards obtained from commercial sources (Merck, Kenilworth, NJ, USA; Fluka, St. Louis, MO, USA).

The chromatographic measurements were performed on an Agilent 1100 Series LC system consisting of a vacuum degassing unit, a binary high pressure pump, a standard automatic sample injector, a column thermostat and a diode array detector (DAD). In order to obtain the lipophilicity indices, the retention time of each compound was measured using five different HPLC columns: RP18 (LiChroCART, Purosphere RP-18e, $3 \mathrm{~mm} \times 125 \mathrm{~mm}, 5 \mu \mathrm{m}$ particle size, carbon load-18\%), C8 (Zorbax, Eclipse XDBC8, $4.6 \mathrm{~mm} \times 150 \mathrm{~mm}, 5 \mu \mathrm{m}$ particle size, carbon load-7.6\%), C16-Amide (Discovery RP-Amide C16, $4.6 \mathrm{~mm} \times 150 \mathrm{~mm}, 5 \mu \mathrm{m}$ particle size, carbon load-11\%), CN100 (Saulentechnik, Lichrosphere, $4 \mathrm{~mm} \times 250 \mathrm{~mm}, 5 \mu \mathrm{m}$ particle size), and pentafluorophenyl (Phenomenex, Kinetex PFP, $2.1 \mathrm{~mm} \times 150 \mathrm{~mm}, 2.6 \mu \mathrm{m}$ particle size). The mobile phase consisted of a mixture of methanol and water $(0.1 \%$ formic acid $)$ in different proportions and the injection volume was $1 \mu \mathrm{L}$ of standard solution $(1 \mathrm{mg} / \mathrm{mL})$.

The retention times were measured at $22^{\circ} \mathrm{C}$ and $37^{\circ} \mathrm{C}$ by the UV detector at $254 \mathrm{~nm}$ and for each solute, the retention factor expressed as $k=\left(t_{r}-t_{0}\right) / t_{0}$, was determined at different proportions of methanol. Then, a plot was made using logk vs. \% methanol (in mobile phase), and the extrapolation to $0 \%$ methanol gave $\log k_{w}$. The dead time was measured for all selected columns using urea and they were as follows: $t_{0}(\mathrm{RP} 18)=0.903 \mathrm{~min}, t_{0}(\mathrm{C} 8)=1.614 \mathrm{~min}, t_{0}(\mathrm{C} 16$-Amide $)=1.275 \mathrm{~min}$, $t_{0}(\mathrm{CN})=2.135 \mathrm{~min}$, and $t_{0}(\mathrm{PFP})=1.766 \mathrm{~min}$. The measurements were carried out at a flow rate of $0.7 \mathrm{~mL} / \mathrm{min}$ for RP18, C8, C16-Amide, CN columns and $0.2 \mathrm{~mL} / \mathrm{min}$ for PFP column. In all cases, five different methanol fractions were used for the extrapolation to $\log k_{w}$.

Additionally, in order to compare the experimental results, some lipophilicity descriptors were calculated using different software, such as Chem 3D Ultra 8.0 (http:/ / www.cambridgesoft.com), Dragon Plus version 5.4 (http://www.talete.mi.it), and ChemDoodle (https://www.chemdoodle. com). Thus, 12 descriptors were calculated as follows (Table S1): ChemDraw Ultra 8.0 provided five $\log \mathrm{P}$ values $\left(\log \mathrm{P}, \mathrm{CLog} \mathrm{P}, \log \mathrm{P}^{\mathrm{C}}\right.$-Crippen's method, $\log \mathrm{P}^{\mathrm{V}}$-Viswanadhan's method, $\log \mathrm{P}^{\mathrm{B}}$-Broto's method) calculated on the basis of fragmental and atom based methods; another four $\log \mathrm{P}$ values were calculated by the software Dragon 5.4 using topological descriptors (MLOGP-Moriguchi's method, MLOGP2-Squared Moriguchi's method, ALOGP-Ghose-Crippen's method, and ALOGP2-Squared Ghose-Crippen's method), and ChemDoodle provided three values (NCNHET, AlogP98, XLogP2).

Chemometrics analyses were performed using the Statistica 8.1 software (StatSoft, Tulsa, OA, USA), and CRRN_DNA_V6_S computer code (Excel extension) developed by K. Heberger et al. was used for ranking and classification of indices [15].

\section{Results and Discussion}

The group of antioxidants investigated in this study includes compounds with very different structures, sizes, and polarities, so it is expected that they have quite different chromatographic behavior. Therefore, the methanol fraction contained in the mobile phase was optimized so that all compounds have retention times between $t_{0}$ (dead time) and a maximum of 15 min so that the 
analysis duration is as short as possible and that the results for different temperatures $\left(22{ }^{\circ} \mathrm{C}\right.$ and $37^{\circ} \mathrm{C}$ ) can be compared. Thus, the fraction of methanol, for which a linear range was obtained for $\log k$, ranged between 50-60\% for the RP18 and CN columns, 60-70\% for the C8 and C16-Amide columns, and $55-65 \%$ for the PFP column; and in all cases an increment of $2.5 \%$ was used to obtain the five specified concentrations. The strong linear dependence of retention parameters through the methanol fraction variance was demonstrated by the values of determination coefficient $\left(R^{2}\right)$ higher than 0.99 in all cases.

Furthermore, by evaluating the profiles of $\mathrm{k}$ and $\log k$ values for all methanol fractions determined for both $22{ }^{\circ} \mathrm{C}$ and $37{ }^{\circ} \mathrm{C}$, the regular changes in retention with increasing methanol ratios were observed in the case of C8, C16-Amide, PFP (except Compound 22), and CN column, except RP18. In the case of the four columns, the $\mathrm{mk}$ and $\mathrm{mlog} k$ parameters were overlapping the intermediate (median) value corresponding to the middle concentration of methanol (Figure S1a-e).

All the specific chromatographic lipophilicity parameters (arithmetic mean of $\mathrm{k}$ and $\log k-\mathrm{m} k$ and m logk, $\log k w, S, \phi_{0}$, scores corresponding to the first principal component obtained by applying PCA to the retention data-PC1/ $k$ and $\mathrm{PC} 1 / \log k$ ) were calculated and considered for all investigated columns at $22{ }^{\circ} \mathrm{C}$ and $37^{\circ} \mathrm{C}$, and the obtained results are presented in Tables S2 and S3. By summary evaluation, it can be observed that at $22^{\circ} \mathrm{C}$ pterostilbene has the highest lipophilicity index for the C8, C16-Amide, and CN columns, pelargonidin for the RP18 column, and procyanidin C1 for the PFP column, while at $37{ }^{\circ} \mathrm{C}$ pterostilbene has the highest lipophilicity index for the RP18, C8, and C16-Amide columns, pelargonidin for the $\mathrm{CN}$ column, and apigenin for the PFP column. Additionally, the lowest lipophilicity index at $22{ }^{\circ} \mathrm{C}$ was found for epigallocatechin gallate on RP18 column, procyanidin $\mathrm{C} 1$ on C8 column, protocatechuic acid on C16-Amide and PFP columns, and chlorogenic acid on CN column, while at $37^{\circ} \mathrm{C}$ the lowest lipophilicity index was found for catechin on RP18 and C16-Amide columns, and procyanidin $\mathrm{C} 1$ on $\mathrm{C} 8, \mathrm{CN}$, and PFP columns.

In order to see how the temperature affects the lipophilicity, we will refer only to the indices $\log k_{w}$ and $\mathrm{m} \log k$. First, matrices of correlation between the data obtained at $22{ }^{\circ} \mathrm{C}$ vs. $37^{\circ} \mathrm{C}$ for all columns, including the computational lipophilicity values, were calculated, and the obtained results are presented in Tables S2 and S3. Accordingly, it can be observed, considering firstly experimental $\log k_{w}$ values for the two temperatures, the higher correlations were obtained for $\mathrm{C} 16(\mathrm{r}=0.969), \mathrm{C} 8(\mathrm{r}=0.983)$, and $\mathrm{CN}(\mathrm{r}=0.828)$. A low correlation was obtained for RP18 ( $\mathrm{r}=0.463)$, and surprisingly a very low negative value resulted for PFP $(\mathrm{r}=-0.042)$. The statistical results concerning the computational lipophilicity descriptors indicate that at $22{ }^{\circ} \mathrm{C}$ the highest correlation were obtained on PFP $(\mathrm{r}=0.918$ with NCNHET, $r=0.873$ with XLogP, and $r=0.855$ with ALogP2) and CN ( $r=0.800$ with CLogP and $\mathrm{r}=0.620$ with $\mathrm{MLog} \mathrm{P})$. On the other hand, at $37^{\circ} \mathrm{C}$ the best correlations were obtained on $\mathrm{CN}$ column ( $r=0.533$ with ALogP98) and RP18 ( $r=0.504$ with CLogP). A high correlation was also found for RP18 column vs. Average value $(r=0.906)$ calculated for all experimental and computational data corresponding to each investigated compound; this value is used also in the Heberger algorithm [15-18], as will be discussed below. In addition, the results in Table S2 illustrate a significant correlation between the results obtained on all columns (with some exceptions in the case of PFP and RP18) and the following computational descriptors: CLogP, MLogP and Average.

The statistical evaluation of the correlation results considering the experimental data estimated as mlogk (Table S3) and the computationally indices showed that there is a high correlation between all experimental lipophilicity indices at the two temperatures excepting the correlations between RP18 and $\mathrm{CN}\left(22\right.$ and $37^{\circ} \mathrm{C} ; \mathrm{r}=0.342$ and $\left.\mathrm{r}=0.239\right)$, PFP at $37^{\circ} \mathrm{C}(\mathrm{r}=0.358)$, and $\mathrm{C} 16$ at $37^{\circ} \mathrm{C}(\mathrm{r}=0.384)$. A significant correlation has been observed between the mlogk values and CLogP $(0.525<\mathrm{r}<0.723)$, MLogP $(0.423<\mathrm{r}<0.679)$. A significant correlation is pointed out (with some exceptions) in the case of Average, ALogP98 and XLogP2. In addition, the correlation between mlogk values at $22{ }^{\circ} \mathrm{C}$ and $37^{\circ} \mathrm{C}$ for PFP becomes highly significant $(\mathrm{r}=0.938)$. The large difference between the correlation coefficients obtained for $\log k_{w}$ and mlogk at the two temperatures in the case of PFP column can be clearly explained by the effect of extrapolation in the first case. The profiles of $\log k_{w}$ and $\log k$ presented in Figures 
S2a-e and S3a-e and the scatterplot of data corresponding to $\log k_{w}$ and $\log k$, respectively, at the two temperatures, clearly illustrate a separate chromatographic behavior of Compound 22 (the outsized molecule with a big number of $\mathrm{OH}$ groups), which appears as a strong outlier (extreme) in the first case (Figure 1a,b). Moreover, the effect of temperature on the considered chemically bonded columns and the chromatographic behavior of the investigated compounds is clearly illustrated by the box and whisker plot depicted in Figure 2. The larger difference is observed in both cases on the RP18 and PFP columns and the small effect on $\mathrm{C} 16$ and $\mathrm{CN}$, two columns with higher polarity. Considering the mlogk values, a distinct difference can be seen between the nonpolar $\mathrm{C} 8$ and $\mathrm{C} 18$ columns (positive effect) and the CN, C16-Amide, and PFP (negative effect in the order $\mathrm{CN}<\mathrm{C} 16$-Amide $<$ PFP). The discrepancies observed in the case of $\log k_{w}$ values can be explained once again by the effect of extrapolation and the different chromatographic behavior of certain compounds $(13,16,18,19$, and 22).

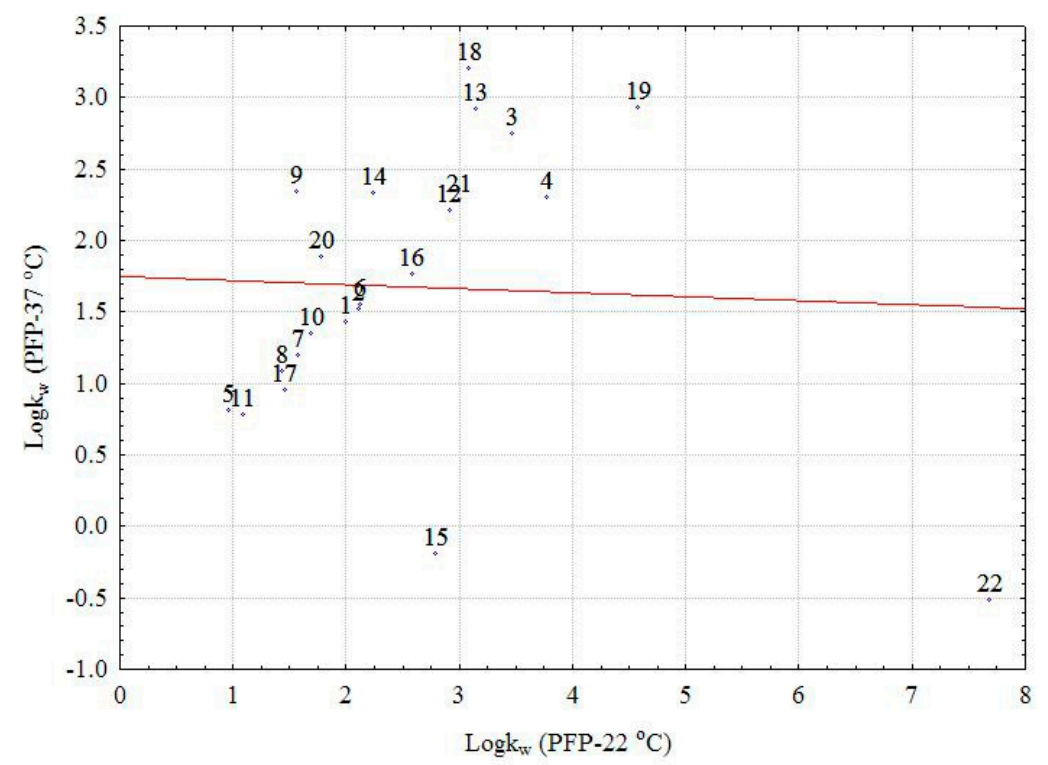

(a)

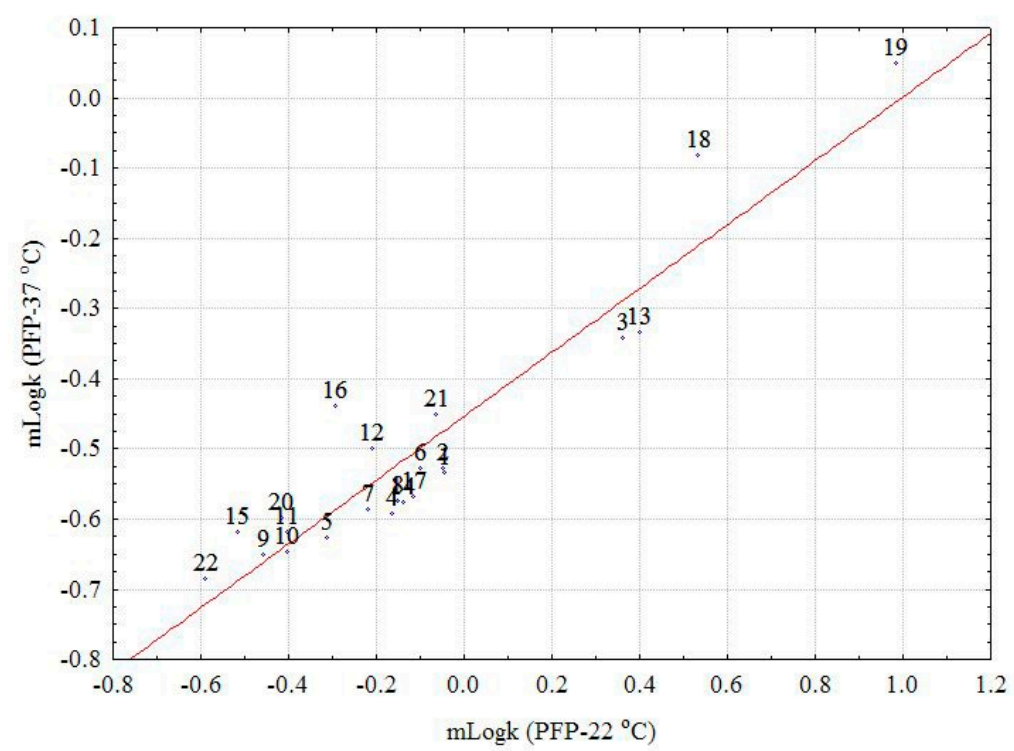

(b)

Figure 1. Scatterplot of $\log k w$ values corresponding to PFP column at $22^{\circ} \mathrm{C}$ (a) and $37^{\circ} \mathrm{C}$; and $\operatorname{mog} k$, respectively (b). 


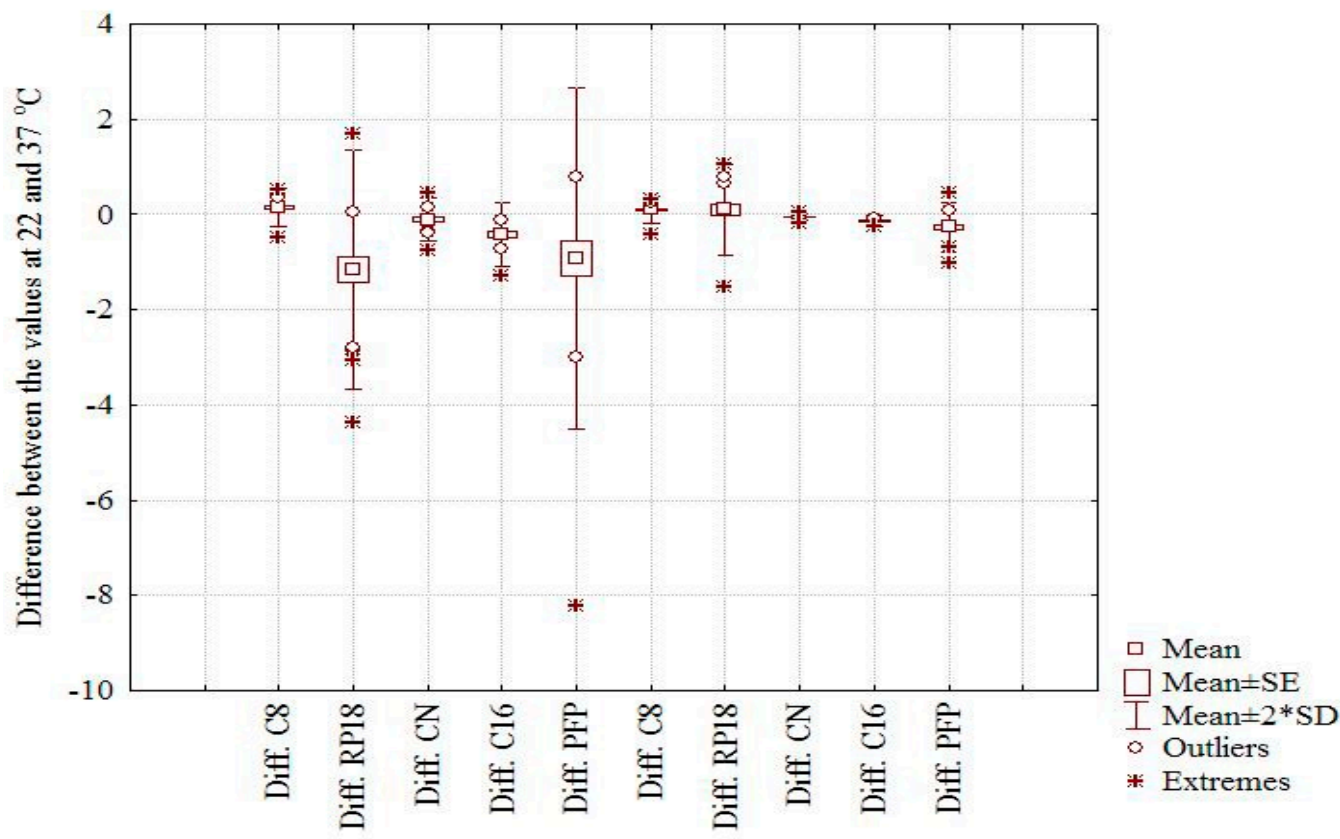

Figure 2. Box and whiskers corresponding to $\log k w$ values, (the first five boxes, from left to right) and mlogk values, respectively (the last five boxes).

The statements above are well supported by the results obtained applying classical hierarchical cluster analysis (HCA) and PCA on the standardized datasets. The dendrogram obtained in the case of dataset including experimental $\log k_{w}$, and computationally indices illustrate three well-separated clusters (Figure S4a). The $\log k_{w}$ corresponding to $\mathrm{CN}$ and $\mathrm{C} 16$ columns at the two temperatures, including MLogP, are in the first group, the second combines the $\log k_{w}$ obtained on C8 at the two temperatures, PFP and RP18 at $37^{\circ} \mathrm{C}$ and some computational indices (ALogP, ALogP98, ClogP, and Average). The third cluster includes $\log k_{w}$ corresponding to PFP and RP18 at $22{ }^{\circ} \mathrm{C}$ and XLogP2, NCNHET, MLogP2, and ALogP2. If the mlogk values are considered, a clear distinction between computationally estimated $\log P s$ and chromatographic indices is obtained. The high similarity of the mlogk is also clearly shown (Figure S4b).

Applying PCA on the $\log k_{w}$ values, the first principal component explains $52.33 \%$ of the total variance, and the second component, $23.90 \%$ : a two-component model thus accounts for $76.23 \%$ of the total variance. The results from the PCA of mlogk values are a little different. The first two PCs account for $75.58 \%$ of the total variance (PC1 54.24\% and PC2 21.34\%). The patterns obtained by two-dimensional representations of the loadings are more or less similar with the HCA-patterns discussed above. In the case of $\log k_{w}$ (Figure S5a), two groups are clearly separated. The first include the majority of the experimental $\log k_{w}$ indices and two computationally scales (ClogP and MLogP), in the second group appear two $\log k_{w}\left(\mathrm{RP} 18-37^{\circ} \mathrm{C}\right.$ and PFP-22 ${ }^{\circ} \mathrm{C}$ ) near the other computationally scales. Two major groups are present also in the case of the mlogk dataset. The first group includes all the mlogk indices and two computational scales (CLogP and MLogP), and in the second group we find only computationally scales (Figure S5b).

At the same time, the lipophilic character similarities existing between the investigated compounds may be illustrated by the lipophilicity charts ("holistic lipophilicity chart") obtained by 2-D scatterplots of the scores corresponding to the first two principal components. The score plots (Figure S6a,b) reveal two groups (more compacted in the case of $\log k_{w}$ ) and identify two outliers: pterostilbene (19) and C1 type proanthocyanidin (22). A two-way joining cluster analysis applied on a dataset formed by the $\log k_{w}$ and mlogk values obtained for all compounds on all investigated columns at the two temperatures including the computationally calculated indices provides similar conclusions regarding the effect of temperature and the chromatographic behavior of the compounds 
investigated (Figure S7a). The most similar results, considering $\log k_{w}$ values and the computational scales, for example, are easily observed in the case of $\mathrm{CN}, \mathrm{C} 8$, and $\mathrm{C} 16$ at the two temperatures (green color), and the outlier position of the C1 type proanthocyanidin (22) (yellow color) is also clearly indicated. The pattern in the case of $\mathrm{m} \log k$ values including the computational scales illustrates a high similarity among all experimentally indices and CLogP, ALogP and Average appear to be closer to them (Figure S7b). In order to get more information and a better understanding of the experimentally and computationally estimation of lipophilicity, we also applied a new non-parametric ranking method, a sum of ranking differences-comparison of ranks by random numbers (SRD-CRRN) [15-18]. According to the SRD-CRRN, considering first the $\log k_{w}$ values and computationally scales, the best descriptors are obtained using PFP-22 ${ }^{\circ} \mathrm{C}, \mathrm{RP} 18-37{ }^{\circ} \mathrm{C}, \mathrm{CN}-22{ }^{\circ} \mathrm{C}$, and $\mathrm{C} 8-22{ }^{\circ} \mathrm{C}$ including ALogP2 (the best), ALogP, and CLogP. Lower ranking values were obtained in the case of RP18-22 ${ }^{\circ} \mathrm{C}, \mathrm{PFP}-22^{\circ} \mathrm{C}$, and MLogP and MLogP2 (Figure 3). In the case of the dataset comprising mlogk values and calculated $\log \mathrm{P}$ values, the results presented in Figure 3 indicate ALogP2, CLogP, and ALogP as the best computationally scales followed by two groups of lipophilicity measures: (CN, C16, and RP18 at $22{ }^{\circ} \mathrm{C}$ and $\left.\mathrm{MLogP}\right)$ and $\left(\mathrm{X} \log \mathrm{P} 2, \mathrm{C} 16\right.$, and $\mathrm{PFP}$ at $37^{\circ} \mathrm{C}, \mathrm{CN}-37^{\circ} \mathrm{C}$, and $\left.\mathrm{C} 8-22{ }^{\circ} \mathrm{C}\right)$. The farthest group includes $\mathrm{C} 8$ and RP18 at $37^{\circ} \mathrm{C}$, as well as MLogP and NCNHET, and they are considered the worst lipophilicity measures.

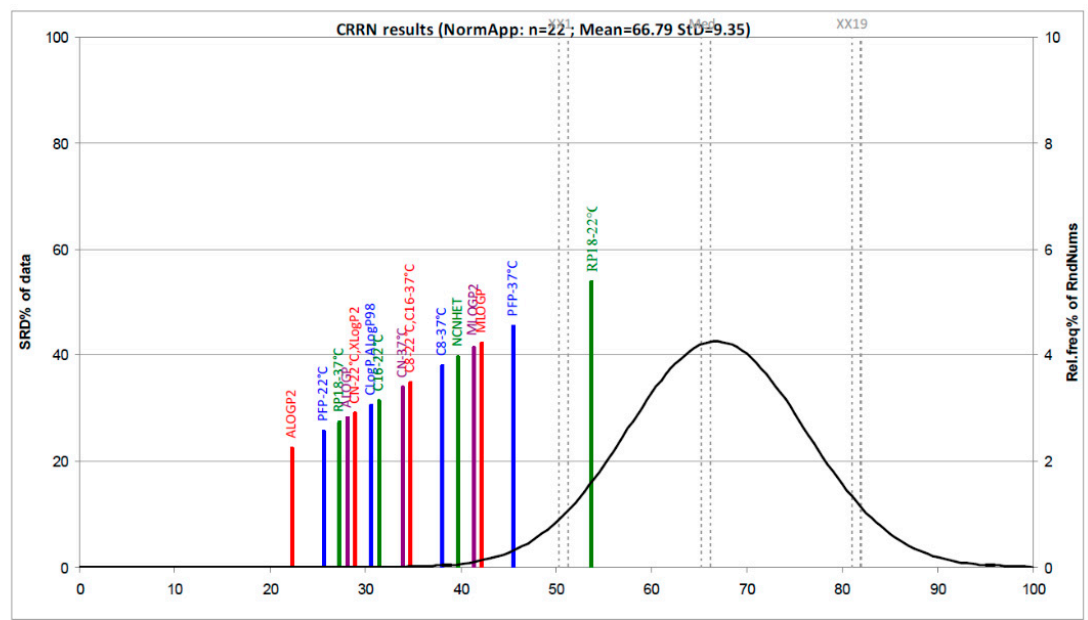

(a)

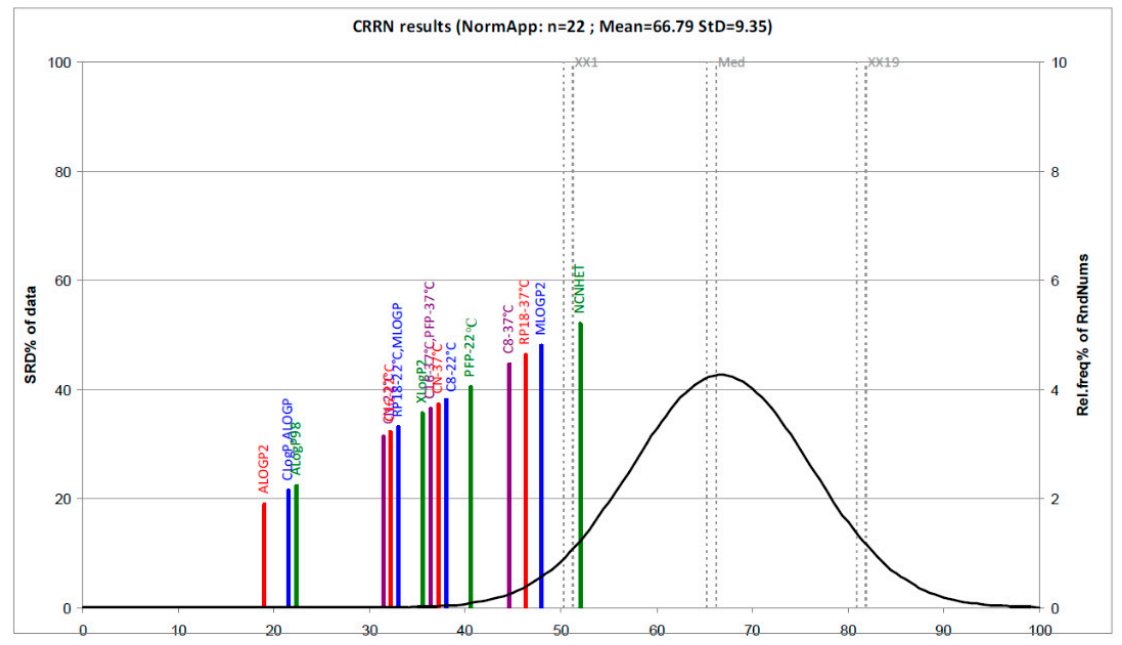

(b)

Figure 3. SRD-CRRN ranking of chromatographically estimated lipophilicity indices logkw (a) and mlogk (b), and computationally calculated $\log P$ values. 


\section{Concluding Remarks}

Investigations concerning the lipophilicity of a group of antioxidant compounds were conducted using reversed phase high-performance liquid chromatography. Different mixtures of methanol-water as mobile phase and several stationary phases, such as RP18, C8, C16-Amide, CN, and PFP were tested, and the results indicated pterostilbene as the most lipophilic compound. Significant correlations were obtained between different experimental indices of lipophilicity at the two temperatures and some computed $\log \mathrm{P}$ scales (CLogP, MLogP, and ALogP98), and the mlogk values were the most correlated with the computed indices. In addition, the results obtained in this study by applying multivariate exploratory techniques, such as HCA, PCA, or the two-way joining clustering and profile representation, illustrated more or less the same (dis)similarities of the stationary phases and were well supported by the ranking scales generated applying SRD-CRRN algorithm. Overall, the results (mainly mlogk indices) illustrate a similar and small effect of temperature on the chromatographic behavior of the investigated compounds in all cases. In consequence, we concluded that the mean $(\mathrm{m} \log k)$ is a better lipophilicity estimator, as it is not affected as much by experimental and model errors like in the case of the extrapolation estimator $\left(\log k_{w}\right)$, a conclusion which was also pointed out in the literature and well supported by these results.

Supplementary Materials: Supplementary materials are available online.

Acknowledgments: Project "Antioxidant Power Series as a tool rational design and assessment of health promoting properties of functional food based on antioxidant phytochemicals" (number of the application 2014/14/A/ST4/00640) financed by National Science Centre, Poland in a programme "MAESTRO 6."

Author Contributions: All authors contributed equally.

Conflicts of Interest: The authors declare no conflict of interest.

\section{References}

1. Rice-Evans, C.; Miller, N.; Bolwell, P.; Bramley, P.; Pridham, J. The relative antioxidant activities of plant-derived polyphenolic flavonoids. Free Rad. Res. 1995, 22, 375-383. [CrossRef]

2. Sangster, J. Octanol-water partition coefficients of simple organic compounds. J. Phys. Chem. Ref. Data 1998, 18, 1111-1229. [CrossRef]

3. Casoni, D.; Kot-Wasik, A.; Namiesnik, J.; Sârbu, C. Lipophilicity data for some preservatives estimated by reversed-phase liquid chromatography and different computation methods. J. Chromatogr. A 2009, 1216, 2456-2465. [CrossRef] [PubMed]

4. Briciu, R.D.; Kot-Wasik, A.; Namiesnik, J.; Sârbu, C. A comparative study of the molecular lipophilcity indices of vitamins $\mathrm{A}$ and $\mathrm{E}$ and of some precursors of vitamin A, estimated by HPLC and by different computation methods. Acta Chromatogr. 2009, 21, 237-250. [CrossRef]

5. Snyder, L.R.; Kirkland, J.J. Introduction to Modern Liquid Chromatography, 2nd ed.; John Wiley and Sons: New York, NY, USA, 1979.

6. Haber, P.; Bauczek, T.; Kaliszan, R.; Snyder, L.R.; Dolan, J.W.; Wehr, C.T. Computer simulation for the simultaneous optimization of any two variables and any chromatographic procedure. J. Chromatogr. Sci. 2000, 38, 386-392. [CrossRef] [PubMed]

7. Valkó, K.; Segel, P. New chromatographic hydrophobicity indices $\left(\phi_{0}\right)$ based on the slope and the intercept of $\log k^{\prime}$ versus organic phase concentration plot. J. Chromatogr. A 1993, 631, 49-61. [CrossRef]

8. Du, C.M.; Valkó, K.; Bevan, C.; Reynolds, D. Rapid gradient RP-HPLC method for lipophilicity determination: A solvation equation based comparison with isocratic methods. Anal. Chem. 1997, 70, 4228-4234. [CrossRef]

9. Briciu, R.D.; Kot-Wasik, A.; Wasik, A.; Namieśnik, J.; Sârbu, C. The lipophilicity of artificial and natural sweeteners estimated by reversed-phase thin-layer chromatography and computed by various methods. J. Chromatogr. A 2010, 1217, 3702-3706. [CrossRef] [PubMed]

10. Casoni, D.; Petre, J.; David, V.; Sârbu, C. Prediction of pesticides chromatographic lipophilicity from the computational molecular descriptors. J. Sep. Sci. 2011, 34, 247-254. [CrossRef] [PubMed]

11. Sârbu, C.; Casoni, D.; Kot-Wasik, A.; Wasik, A.; Namieśnik, J. Modeling of chromatographic lipophilicity of food synthetic dyes estimated on different columns. J. Sep. Sci. 2010, 33, 2219-2229. [CrossRef] [PubMed] 
12. Sârbu, C.; Naşcu-Briciu, R.D.; Casoni, D.; Kot-Wasik, A.; Wasik, A.; Namieśnik, J. Chromatographic lipophilicity determination using large volume injections of the solvents non-miscible with the mobile phase. J. Chromatogr. A 2012, 1266, 53-60. [CrossRef] [PubMed]

13. Tetko, I.V.; Gasteiger, J.; Todeschini, R.; Mauri, A.; Livingstone, D.; Ertl, P.; Palyulin, V.A.; Radchenko, E.V.; Zefirov, N.S.; Makarenko, A.S.; et al. Virtual Computational Chemistry Laboratory-Design and Description. J. Comput. Aided Mol. Des. 2005, 19, 453-463. [CrossRef] [PubMed]

14. Mannhold, R.; Poda, G.I.; Ostermann, C.; Tetko, I.V. Calculation of Molecular Lipophilicity: State-of-the-Art and Comparison of Log P Methods on More Than 96,000 Compounds. J. Pharm. Sci. 2009, 98, 861-893. [CrossRef] [PubMed]

15. Héberger, K. Sum of ranking differences compares methods or models fairly. Trends Anal. Chem. 2010, 29, 101-109. [CrossRef]

16. Andric, F.; Heberger, K. Chromatographic and computational assessment of lipophilicity using sum of ranking differences and generalized pair-correlation. J. Chromatogr. A 2015, 1380, 130-138. [CrossRef] [PubMed]

17. Andric, F.; Heberger, K. Towards better understanding of lipophilicity: Assessment of in silico and chromatographic $\log \mathrm{P}$ measures for pharmaceutically important compounds by nonparametric rankings. J. Pharm. Biomed. Anal. 2015, 115, 183-191. [CrossRef] [PubMed]

18. Andric, F.; Bajusz, D.; Racz, A.; Segan, S.; Heberger, K. Multivariate assessment of lipophilicity scales-Computational and reversed phase thin-layer chromatographic indices. J. Pharm. Biomed. Anal. 2016, 127, 81-93. [CrossRef] [PubMed]

Sample Availability: Not available.

(C) 2017 by the authors. Licensee MDPI, Basel, Switzerland. This article is an open access article distributed under the terms and conditions of the Creative Commons Attribution (CC BY) license (http:/ / creativecommons.org/licenses/by/4.0/). 